\title{
A Rare Case of Adenosquamous Carcinoma Arising in the Background of IgG4-Related Lung Disease
}

Sangjoon Choi · Sujin Park

Man Pyo Chung ${ }^{1}$. Tae Sung Kim²

Jong $\mathrm{Ho} \mathrm{Cho}^{3} \cdot$ Joungho Han

Departments of Pathology and Translational Genomics, IInternal Medicine, ${ }^{2}$ Radiology, and

${ }^{3}$ Thoracic and Cardiovascular Surgery,

Samsung Medical Center, Sungkyunkwan

University School of Medicine, Seoul, Korea

Received: December 3, 2018

Revised: January 26, 2019

Accepted: February 20, 2019

\section{Corresponding Author}

Joungho Han, MD

Department and of Pathology and Translational

Genomics, Samsung Medical Center,

Sungkyunkwan University School of Medicine,

81 Irwon-ro, Gangnam-gu, Seoul 06351, Korea

Tel: +82-2-3410-2800

Fax: +82-2-3410-0025

E-mail: hanjho@skku.edu
lgG4-related disease is a systemic inflammatory disease and is known as IgG4-related lung disease (lgG4-RLD) when it involves the respiratory system. Primary lung cancer arising from a background of IgG4-RLD is very rare. Herein, we report a case of adenosquamous carcinoma arising from the background of IgG4-RLD and presenting as an interstitial lung disease pattern. A 66 -year-old man underwent lobectomy under the impression of primary lung cancer. Grossly, the mass was ill-defined and gray-tan colored, and the background lung was fibrotic. Microscopically, tumor cells showed both squamous and glandular differentiation. Dense lymphoplasmacytic infiltration with fibrosis and obliterative phlebitis were seen in the background lung. IgG4 immunohistochemical stain showed diffuse positivity in infiltrating plasma cells. Primary lung adenosquamous carcinoma has not been reported in a background of IgG4-RLD. Due to the rarity of lgG4-RLD, physicians must follow patients with lgG4-RLD over long periods of time to accurately predict the risk of lung cancer.

Key Words: Lung; Neoplasm; IgG4-related disease; Autoimmune
IgG4-related disease (IgG4-RD) is a rare, chronic, systemic inflammatory disease with increased serum $\operatorname{IgG} 4$ levels that is characterized by dense lymphoplasmacytic infiltration, storiform fibrosis, and obliterative phlebitis with IgG4 immunoreactivity in plasma cells. ${ }^{1}$ When IgG4-RD involves the lung, it is called IgG4-related lung disease (IgG4-RLD) and manifests in various histologic forms, such as solid nodular type, bronchovascular type, or interstitial lung disease type. ${ }^{2-4}$ The association of lung cancer with IgG4-RLD remains unclear, and only a small number of adenocarcinoma-associated cases have been reported. ${ }^{4-6}$ Herein, for the first time, we report a case of adenosquamous carcinoma in a 66-year-old male patient who was followed-up for IgG4-related cholangitis.

\section{CASE REPORT}

A 66-year-old man who had a past medical history of idiopathic pulmonary fibrosis (IPF) and mass-forming IgG4-related autoimmune cholangitis was admitted to the hospital for a newly-identified consolidative lung mass discovered during follow-up. Chest computed tomography revealed a subpleural nodule in the left lower lobe of the lung in a background of reticular and honeycomb fibrosis (Fig. 1A). ${ }^{18} \mathrm{~F}$-fluorodeoxyglucose uptake was detected in the subpleural nodule (Fig. 1B). The results of the pulmonary function tests were within normal range: forced vital capacity (FVC) $3.23 \mathrm{~L}$ ( $82 \%$ of the predicted value), forced expiratory volume in 1 second $\left(\mathrm{FEV}_{1}\right) 2.35 \mathrm{~L}$ (80\% of the predicted value), and $\mathrm{FEV}_{1} / \mathrm{FVC} 73 \%$. Laboratory test showed an increased serum IgG4 level $(232.4 \mathrm{mg} / \mathrm{dL})$. The patient underwent lobectomy under the impression of lung cancer. Grossly, the tumor was ill-defined, gray-tan colored and measured 3.5 ×3.2 $\times 2.0$ $\mathrm{cm}$. The background lung was fibrotic and emphysematous (Fig. 1C). Microscopically, the background lung showed diffuse irregular interstitial fibrosis with dense lymphoplasmacytic infiltration and occasional obliterative phlebitis (Fig. 1D-F). Tumor cells showed both squamous and glandular differentiation. The squamous cell carcinoma component was composed of moderately to poorly differentiated tumor cells that contained keratin 

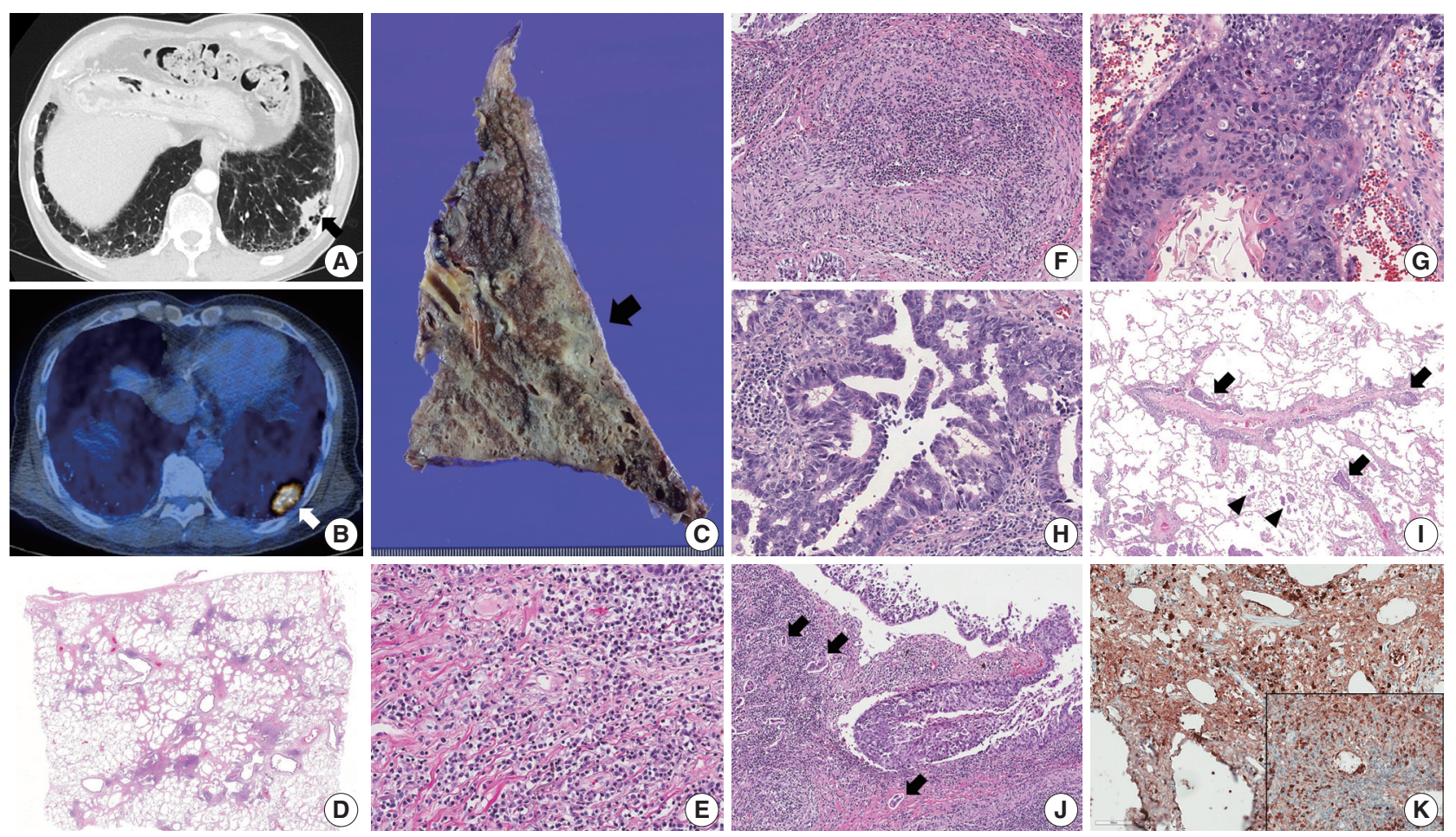

Fig. 1. (A, B) Chest computed tomography shows a consolidative nodule (arrow) in a background of subpleural reticulation and honeycomb fibrosis at both lung bases. Positron emission tomography reveals ${ }^{18} \mathrm{~F}$-fluorodeoxyglucose uptake by the nodule. (C) The cut section of the lung showed an ill-defined and gray-tan colored mass (arrow). The background lung was emphysematous and fibrotic. (D-F) Histologic examination shows irregular interstitial fibrosis with patchy lymphoid aggregation, predominant lymphoplasmacytic infiltration, and occasional obliterative phlebitis. (G) The squamous cell carcinoma component shows keratinization and multifocal dyskeratosis. $(H)$ The adenocarcinoma component was mainly composed of a moderately differentiated acinar pattern. (I) Diffuse spread through air space (arrowheads) and multifocal lymphangitic spread (arrow) of tumor cells are frequently found at the periphery of the mass. (J) Dense fibrosis and lymphoplasmacytic infiltration are found in the peritumoral area. Multifocal endolymphatic tumor emboli (arrows) are also noted. (K) Both lgG4 and lgG (inset) immunohistochemical stains show diffuse positivity in the infiltrating plasma cells. The lgG4/lgG ratio was over 40\%.

pearls (Fig. 1G). The glandular component was mainly acinar pattern with focal micropapillary pattern (Fig. 1H). Diffuse spread through air space of tumor cells was frequently found at the periphery of the mass (Fig. 1I). Multifocal lymphangitic spreading of tumor cells and metastatic lymph nodes were found (Fig. 1I). Dense fibrosis and lymphoplasmacytic infiltration were adjacent to the tumor cells (Fig. 1J). The final pathologic stage was pT2aN2M0 by the American Joint Committee on Cancer seventh staging system. Immunohistochemistry (IHC) staining revealed the squamous cell carcinoma component was focally positive for p63 (1:200, Biocare, Concord, CA, USA), and the glandular component was negative for TTF-1 (1:50, Dako, Glostrup, Denmark). Additional tests for anaplastic lymphoma kinase (ALK) IHC staining (1:40, NCL-ALK, clone 5A4, Novocastra, Newcastle upon Tyne, UK) and epidermal growth factor receptor gene mutation analysis using a PNA clamping kit (Panagene, Inc., Daejeon, Korea) were negative, and up to $10 \%$ of the tumor cells showed membrane positivity for programmed death- ligand 1 (RTU, 22C3, Dako). IgG4 (1:2,000, The Binding Site, Birmingham, UK) IHC stain showed diffuse positivity in infiltrating plasma cells ( $>50$ cells/high-power field), and the IgG4/ IgG ratio was over $40 \%$ (Fig. 1K). Thus, the patient's IPF was thought to be a manifestation of IgG4-RLD, and we concluded that primary adenosquamous carcinoma had developed in the background of IgG4-RLD. This study was approved by the Institutional Review Board of the Samsung Medical Center with a waiver of informed consent (IRB No. 2018-11-053) and performed in accordance with the principles of the Declaration of Helsinki.

\section{DISCUSSION}

IgG4-RD was first reported as autoimmune pancreatitis in 2001. IgG4-RD is known to predominantly involve the pancreas, hepatobiliary tract, salivary glands, and lacrimal glands, and lung or pleural involvement can occur in up to $35 \%$ of patients. ${ }^{8}$ The 
Table 1. Clinicopathologic findings of previously reported cases of concurrent lgG4-RLD lung cancer

\begin{tabular}{|c|c|c|c|c|c|c|c|c|c|c|}
\hline Reference & Sex & $\begin{array}{l}\text { Age } \\
\text { (yr) }\end{array}$ & Location & $\begin{array}{l}\text { Type of } \\
\text { tumor }\end{array}$ & Pattern of ADC & Radiologic finding & $\begin{array}{l}\text { Pattern of } \\
\text { lgG4-RLD }\end{array}$ & TNM stage & $\begin{array}{l}\text { Other } \\
\text { manifestations }\end{array}$ & $\begin{array}{l}\text { Serum lgG4 } \\
\text { (mg/dL) }\end{array}$ \\
\hline Present case & $\mathrm{M}$ & 66 & LLL & ASC & $\begin{array}{l}\text { Acinar and focal } \\
\text { micropapillary }\end{array}$ & $\begin{array}{l}\text { Subpleural nodule in a } \\
\text { background of reticular and } \\
\text { honeycomb fibrosis }\end{array}$ & Interstitial & pT2aN2M0 & $1 \mathrm{HD}$ & 232 \\
\hline Zen et al. ${ }^{4}$ & M & NA & $R L L$ & ADC & $\begin{array}{l}\text { Mixed, including } \\
\text { acinar }\end{array}$ & $\begin{array}{l}\text { Nodular lesion within the reticular } \\
\text { shadow }\end{array}$ & Interstitial & pT1N2M0 & No & NA \\
\hline Inoue et $a .^{5}$ & M & 78 & RUL & ADC & Lepidic & $\begin{array}{l}\text { Ground-glass opacity with central } \\
\text { collapse and pleural indentation }\end{array}$ & Nodular & pT1bNOMO & Pancreas & 983 \\
\hline Tashiro et al. ${ }^{6}$ & M & 72 & RML & ADC & Lepidic & $\begin{array}{l}\text { Spiculated nodule with pleural } \\
\text { indentation }\end{array}$ & Nodular & pT1bNOM0 & No & 346 \\
\hline
\end{tabular}

IgG4-RLD, IgG4-related lung disease; ADC, adenocarcinoma; M, male; LLL, left lower lobe; ASC, adenosquamous carcinoma; Interstitial, interstitial lung disease type; IHD, intrahepatic bile duct; RLL, right lower lobe; NA, not available; RUL, right upper lobe; Nodular, solid nodular type; RML, right middle lobe.

histologic patterns of IgG4-RLD are divided into three types: solid nodular type, bronchovascular type, and interstitial lung disease type. $^{4}$

It is still debatable whether IgG4-RD is associated with malignancy. Yamamoto et al. ${ }^{9}$ observed 106 IgG4-RD patients (primarily with Mikulicz's disease), and the high standardized incidence rate (SIR) of 3.83 supported the association between IgG4-RD and increased incidence of total malignancies. In a different study, Hirano et al. ${ }^{10}$ observed 113 patients with IgG4$\mathrm{RD}$ (primarily with autoimmune pancreatitis), and the SIR was not significant (1.04). These different outcomes likely result from whether the studies considered cases that simultaneously found malignancies and IgG4-RD.

However, none of these studies included patients with IgG4$\mathrm{RD}$ that involved the pulmonary system. The association of IgG4-RLD with lung cancer has not been studied, and only three lung cancer cases in IgG4-RLD patients have been reported with their histopathologic findings. ${ }^{4-6}$

The patient in the present case had primary adenosquamous carcinoma, which has not been reported alongside IgG4-RD in the previous literature. The adenosquamous carcinoma was characterized by poorly differentiated squamous and glandular tumor cells with lymph node metastases, and the IgG4-RLD background presented as interstitial lung disease. Inoue et al. ${ }^{5}$ and Tashiro et al. ${ }^{6}$ reported a well differentiated lepidic pattern of adenocarcinoma accompanied by IgG4-RLD as a solid nodule or ground glass opacity pattern. There were no lymph node metastases in these two cases. Zen et al. . $^{4}$ reported a moderately differentiated, mixed pattern (including acinar pattern) adenocarcinoma in a background of IgG4-RLD presenting as interstitial pneumonia. Lymph node metastases were found, and the pathologic stage was pT1N2M0. In the present case, similar to Zen's report, moderately to poorly differentiated carcinoma occurred in a background of IgG4-RLD with an interstitial lung disease pat- tern. Numerous lymphovascular invasions and lymph node metastases were found, and the final pathologic stage was pT2aN2M0. Table 1 summarizes the clinicopathologic and radiological characteristics of the reported cases of concurrent IgG4-RLD and lung cancer.

There have been no studies on whether IgG4-RLD increases the risk of malignancy. Although there have been a small number of cases, it is likely that lung cancer more frequently occurs in the solid nodular or interstitial lung disease type of IgG4-RLD rather than the bronchovascular type. Thus far, malignancy has not been reported in the bronchovascular type of IgG4-RLD. There is also a possibility that the differentiation or aggressiveness of the tumor may depend on the background type of IgG4RLD, and the prognosis could be worse in patients with the interstitial lung disease background. Further studies with more cases are needed to elucidate the relationship between tumor aggressiveness and patterns of IgG4-RLD.

\section{ORCID}

Sangjoon Choi: https://orcid.org/0000-0003-2108-0575

Sujin Park: https://orcid.org/0000-0001-7819-5678

Man Pyo Chung: https://orcid.org/0000-0002-5548-0764

Tae Sung Kim: https://orcid.org/0000-0001-7512-0283

Jong Ho Cho: https://orcid.org/0000-0003-3362-4621

Joungho Han: https://orcid.org/0000-0003-4424-7008

\section{Author Contributions}

Conceptualization: SC.

Data curation: SC, JH.

Formal analysis: SC, SP.

Investigation: SC, SP.

Methodology: SC, SP, JH.

Project administration: SC, JH. 
Resources: MPC, TSK, JHC.

Supervision: JH.

Validation: JH.

Writing—original draft: SC, JH.

Writing—review \& editing: SC, SP, JH.

\section{Conflicts of Interest}

The authors declare that they have no potential conflicts of interest.

\section{REFERENCES}

1. Deshpande V, Zen Y, Chan JK, et al. Consensus statement on the pathology of IgG4-related disease. Mod Pathol 2012; 25: 1181-92.

2. Ahn JH, Hong SI, Cho DH, Chae EJ, Song JS, Song JW. A case of IgG4-related lung disease presenting as interstitial lung disease. Tuberc Respir Dis 2014; 77: 85-9.

3. Cho DH, An JH, Kang YM, Chae EJ, Song JS, Song JW. A case of IgG4-related lung disease mimicking non-specific interstitial pneumonia. Korean J Med 2015; 88: 308-12.
4. Zen Y, Inoue D, Kitao A, et al. IgG4-related lung and pleural disease: a clinicopathologic study of 21 cases. Am J Surg Pathol 2009; 33: 1886-93.

5. Inoue T, Hayama M, Kobayashi S, et al. Lung cancer complicated with IgG4-related disease of the lung. Ann Thorac Cardiovasc Surg 2014; 20 Suppl: 474-7.

6. Tashiro H, Takahashi K, Nakamura T, Komiya K, Kimura S, Sueoka-Aragane N. Coexistence of lung cancer and immunoglobulin G4-related lung disease in a nodule: a case report. J Med Case Rep 2016; 10: 113.

7. Hamano H, Kawa S, Horiuchi A, et al. High serum IgG4 concentrations in patients with sclerosing pancreatitis. N Engl J Med 2001; 344: $732-8$

8. Fei $Y$, Shi J, Lin W, et al. Intrathoracic involvements of immunoglobulin G4-related sclerosing disease. Medicine (Baltimore) 2015; 94: e2150.

9. Yamamoto M, Takahashi H, Tabeya T, et al. Risk of malignancies in IgG4-related disease. Mod Rheumatol 2012; 22: 414-8.

10. Hirano K, Tada M, Sasahira N, et al. Incidence of malignancies in patients with IgG4-related disease. Intern Med 2014; 53: 171-6. 